\title{
The GEO 600 laser system
}

\author{
I Zawischa ${ }^{1}$, M Brendel ${ }^{1}$, K Danzmann ${ }^{2,3}$, C Fallnich $^{1}$, M Heurs ${ }^{2}$, \\ S Nagano ${ }^{3}$, V Quetschke ${ }^{2}$, H Welling ${ }^{1}$ and B Willke ${ }^{2,3}$ \\ ${ }^{1}$ Laser Zentrum Hannover, Hollerithallee 8, D-30419 Hannover, Germany \\ ${ }^{2}$ Universität Hannover, Institut für Atom- und Molekülphysik, Callinstraße 38, \\ D-30167 Hannover, Germany \\ ${ }^{3}$ Max-Planck-Institut für Gravitationsphysik, Albert-Einstein-Institut, Außenstelle Hannover, \\ Callinstraße 38, D-30167 Hannover, Germany \\ E-mail: za@1zh.de
}

Received 3 October 2001

Published 18 March 2002

Online at stacks.iop.org/CQG/19/1775

\begin{abstract}
Interferometric gravitational wave detectors require high optical power, single frequency lasers with very good beam quality and high amplitude and frequency stability as well as high long-term reliability as input light source. For GEO 600 a laser system with these properties is realized by a stable planar, longitudinally pumped $12 \mathrm{~W} \mathrm{Nd:YAG} \mathrm{rod} \mathrm{laser} \mathrm{which} \mathrm{is} \mathrm{injection-locked} \mathrm{to} \mathrm{a} \mathrm{monolithic}$ $800 \mathrm{~mW}$ Nd:YAG non-planar ring oscillator. Frequency control signals from the mode cleaners are fed to the actuators of the non-planar ring oscillator which determines the frequency stability of the system. The system power stabilization acts on the slave laser pump diodes which have the largest influence on the output power. In order to gain more output power, a combined Nd:YAG$\mathrm{Nd}: \mathrm{YVO}_{4}$ system is scaled to more than $22 \mathrm{~W}$.
\end{abstract}

PACS numbers: $0480 \mathrm{~N}, 9555 \mathrm{Y}$

\section{Introduction}

The sensitivity of interferometric gravitational wave detectors fundamentally depends on the light power circulating in the interferometer arms. For the GEO 600 detector about $10 \mathrm{~kW}$ of circulating power is needed. Taking into account the finesse of the power recycling cavity and losses in the input optics, an estimated laser output power of $10 \mathrm{~W}$ is required.

In the Fourier frequency band of interferometer sensitivity to gravitational waves, asymmetries and light scattering in the interferometer require a high power and frequency stability at the input light. At the interferometer input in front of the power recycling mirror the most stringent specifications are given in a frequency range between $10 \mathrm{~Hz}$ and $100 \mathrm{~Hz}$. In this Fourier band the frequency fluctuations of the input light with respect to the power recycling cavity should not exceed $2 \times 10^{-4} \mathrm{~Hz} \mathrm{~Hz}^{-1 / 2}$ and amplitude fluctuations should stay 


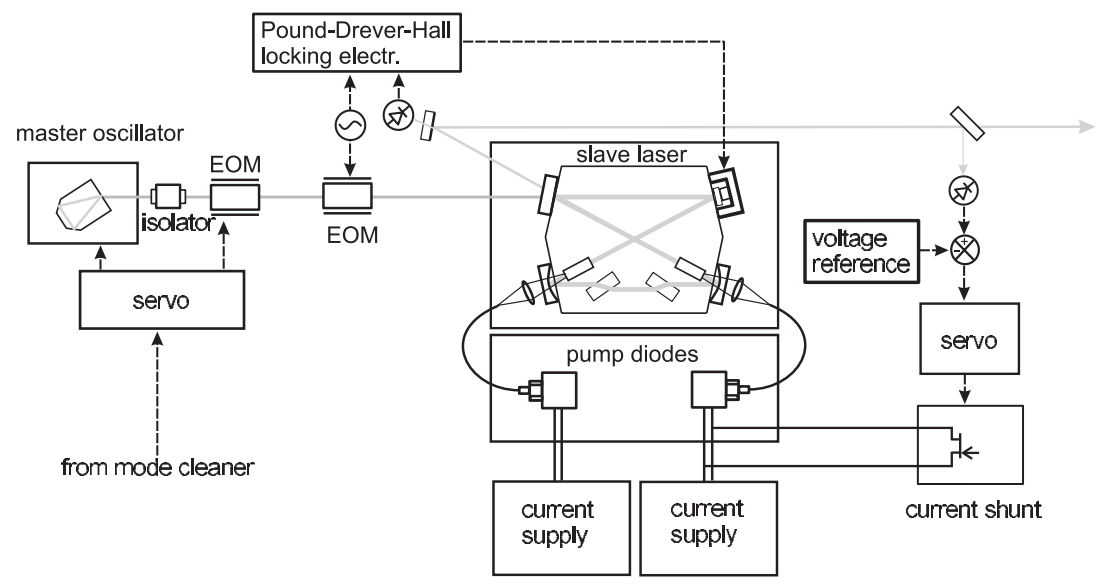

Figure 1. Scheme of the GEO 600 injection-locked laser system.

smaller than $5 \times 10^{-8} \mathrm{~Hz}^{-1 / 2}$ [1]. In order to achieve these demanding requirements, a $12 \mathrm{~W}$ injection-locked laser with very good passive stability was developed and active control loops were implemented.

In the following we will give an overview of the laser system including the passive stability features, active control actuators and a first power pre-stabilization loop. The frequency stabilization loops are described elsewhere in this volume [2]. An approach to increase laser efficiency and output power is described in the last section.

\section{Injection-locked lasers}

In order to supply the required input light to the GEO 600 interferometer an injection-locked laser system [3] was constructed (figure 1). The radiation of a stable single frequency laser (master oscillator) is injected into a power oscillator (slave laser). As soon as the resonance of the power oscillator cavity deviates from the master frequency by less than the so-called locking range $\Delta v=T_{\mathrm{oc}} \mathrm{FSR} /(2 \pi) \sqrt{P_{\text {master }} / P_{\text {slave }}}$, the slave laser emission is synchronized with the master oscillator. If the deviation from exact resonance is kept small compared to the locking range the reduced influence of the slave's eigenfrequency fluctuations $\delta v_{\text {slave }}$ to the system output frequency can be expressed in terms of their Fourier frequency $f,\left|\delta v_{\text {system }}(f) / \delta v_{\text {slave }}(f)\right|=1 / \sqrt{1+(\Delta v / f)^{2}}$ [4-7]. At Fourier frequencies much smaller than the locking range, the influence of the slave is strongly suppressed and the system stability is determined by the master laser.

Due to the saturation of the slave laser's gain, the power noise at low Fourier frequencies (the interferometer detection band) is mainly determined by the slave laser's pump source and must hence be controlled at the latter or in the high power output beam $[8,9]$.

\section{Master laser}

For GEO 600 a diode-pumped monolithic miniature Nd:YAG non-planar ring-laser (NPRO) [10-12] (Innolight Mephisto $800 \mathrm{NE}$ ) with $800 \mathrm{~mW}$ output at $1064 \mathrm{~nm}$ wavelength is used as the master oscillator. This type of laser is well known for its high passive stability and good 


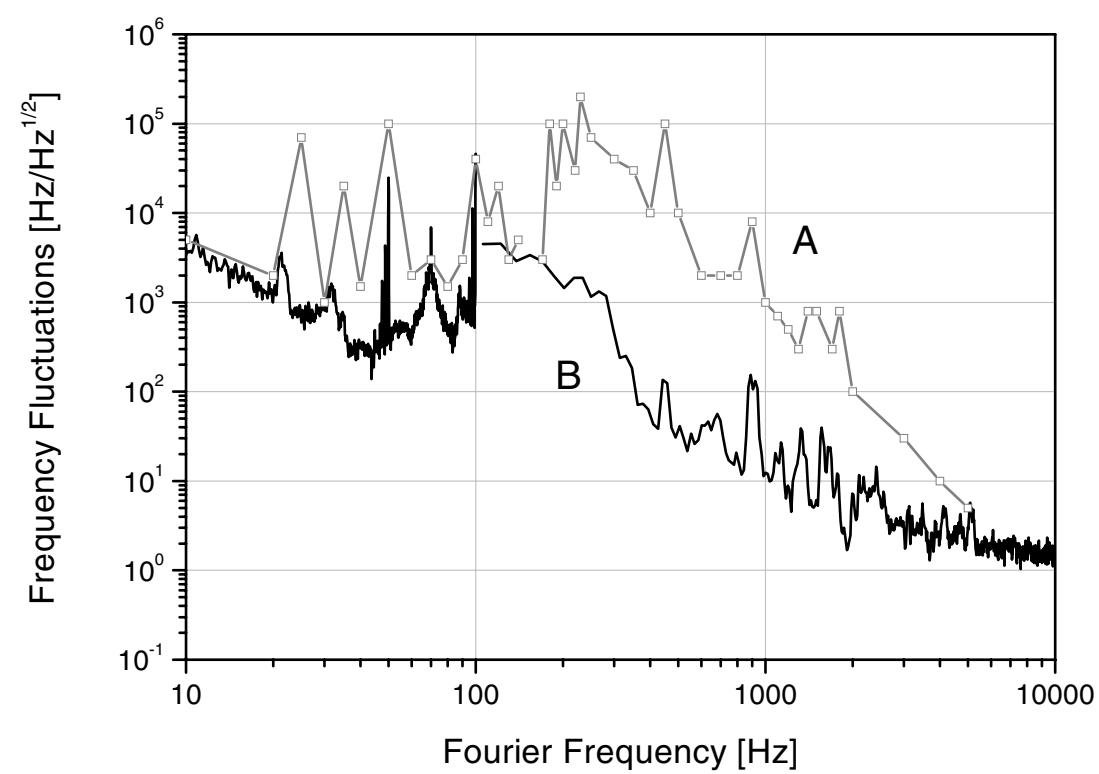

Figure 2. Spectral density of the eigenfrequency fluctuations of the slave laser cavity. The data are derived from the piezo length actuator signal that is necessary to keep the slave laser exactly in resonance with a frequency-stabilized master oscillator. (A) Conventional laser resonator assembled from multiple discrete components. (B) Quasi-monolithic slave laser resonator.

premises for further active stabilization $[13,14]$. The frequency correction signals from the mode cleaners are fed to a piezo ceramic actuator and a Peltier element, which are attached directly to the NPRO crystal and change the laser frequency by means of stress induction and temperature change. For phase correction with Fourier frequencies above $50 \mathrm{kHz}$, an external electro-optic modulator (EOM) is set in the master output beam. Alternatively, stabilization can be achieved by a feedback loop to the pump diode current. A bandwidth of $100 \mathrm{kHz}$ has been achieved so far [15].

\section{Slave laser}

A planar bow-tie ring-resonator with two end-pumped Nd:YAG rods serves as the slave laser for GEO $600[1,16]$. So far two prototypes and five serial versions of the laser with the same optical layout have been assembled and brought to operation. At $2 \times 17 \mathrm{~W}$ pump power from the fibre-coupled lasers diodes, these lasers reach an output power of $12-13.5 \mathrm{~W}$ in a TEM 00 mode. The output beams are typically about 1.1 times diffraction limited and have a degree of polarization better than $98 \%$.

Apart from a cable feed through in early copies of the laser the quasi-monolithic resonator spacer and the mirrors - of which three are directly glued to the spacer-seal the resonator cavity such that acoustic noise cannot easily disturb the phase of the circulating field and air borne pollution of the cavity is reduced. At around $100-1000 \mathrm{~Hz}$ this quasi-monolithic design [16] reduces the influence of laboratory noise on the laser by two decades as compared to a laser assembled from discrete components (figure 2). A low sensitivity of the slave resonator to temperature changes is achieved by the choice of Invar steel (Ni36Fe64/1.3912, $\alpha_{l}=0.8 \times 10^{-6} \mathrm{~K}^{-1}$ ) for the resonator block. The overall thermal expansion including the 
effects of the air at constant pressure in the resonator, the Brewster plates, the piezo and the piezo mount was calculated to be $8.6 \times 10^{-7} \mathrm{~m} \mathrm{~K}^{-1}$ for the early versions of the laser resonator where the piezo mount was not made from Invar. The expansion for the later full Invar versions is calculated to be $2.4 \times 10^{-7} \mathrm{~m} \mathrm{~K}^{-1}$. As long as the cavity volume is connected to the environmental air the atmospheric pressure variations change the optical path length in the resonator via the pressure-dependent refractive index of air. A literature value for refractive index of air yields about $80 \mathrm{MHz} \mathrm{hPa}^{-1}$ [17], which corresponds for the slave laser cavity to $1.6 \times 10^{-7} \mathrm{~m} \mathrm{hPa}^{-1}$. The piezo actuator of the slave laser has a range of approximately $4 \mu \mathrm{m}(-100 \mathrm{~V}$ to $500 \mathrm{~V})$. This range accomplishes compensation for temperature fluctuations of the cavity of $\pm 4.5 \mathrm{~K}( \pm 16 \mathrm{~K})$ or pressure fluctuations of $\pm 25 \mathrm{hPa}$. A control loop bandwidth of up to $20 \mathrm{kHz}$ can be achieved.

Currently, investigations on hermetically airtight resonators are underway. The advantage of an airtight resonator is the independence of atmospheric pressure changes. Alternatively, a controlled pressure variation could be used as the length actuator. First tests have shown that deliberate slow pressure variations cause at least three times less beam jitter than the typical piezo systems, which tilt about 5-10 $\mu \mathrm{rad} \mu \mathrm{m}^{-1}$.

\section{System performance}

The single frequency output power of the injection-locked systems is about the sum of the combined master and slave laser powers, i.e. $12.8-14.5 \mathrm{~W}$. With the copy that was implemented at the interferometer site a visibility of $96 \%$ has been achieved at the first mode cleaner. This demonstrates a high compatibility of the laser mode with passive stable resonators.

An injection lock servo based on the Pound-Drever-Hall technique with $10 \mathrm{kHz}$ control bandwidth reduces the rms $(1 \mathrm{~Hz}$ to $100 \mathrm{kHz})$ deviations of the slave laser cavity from the master frequency to $500 \mathrm{~Hz}$, which is $3 \times 10^{-4}$ times the locking range of about $1.5 \mathrm{MHz}$. No detrimental influence of the slave on the system frequency stability has been observed so far.

In order to automatically achieve injection lock, an autolock servo was implemented in the laser control system. The autolock detects a loss of lock by a laser output power drop because in this case the slave is not lasing unidirectional anymore but emits a part of the power in either output direction. Lock acquisition can simply be achieved by the application of a voltage ramp to the slave piezo length transducer until a transient lock is found and the servo can be switched back to normal operation. Typically about five seconds are needed to acquire stable injection lock.

After several months of laboratory tests a copy of the laser was brought to the detector site in January 2001. Until September 2001 only a small power drop of 5\% was observed. It is likely to be caused by a power drop and a spectral change of the pump laser diodes.

Recently, a first electronic intensity noise suppression servo has been implemented. For this purpose about $12 \mathrm{~mW}$ of the laser output are coupled from the main beam onto an InGaAs-PIN photoreceiver (figure 1, right-hand side). The output voltage of the receiver is then compared with a stable reference voltage. The resulting error signal is suitably amplified and fed back to a shunt transistor across one of the slave's pump diode current supplies. The servo bandwidth reaches from DC to about $50 \mathrm{kHz}$. At Fourier frequencies below $2 \mathrm{kHz}$ the residual relative power noise detected in the loop is reduced below $10^{-8} \mathrm{~Hz}^{-1 / 2}$ (figure 3).

The lowest power noise observed below $50 \mathrm{kHz}$ with an independent photoreceiver outside the control loop is about $2 \times 10^{-8} \mathrm{~Hz}^{-1 / 2}$. At the current stage of development disturbances at the AC power line frequency and multiples are still observable in the out-of-loop signal. Further disturbances between $90 \mathrm{~Hz}$ and $2 \mathrm{kHz}$ are likely to be introduced by beam jitter on 


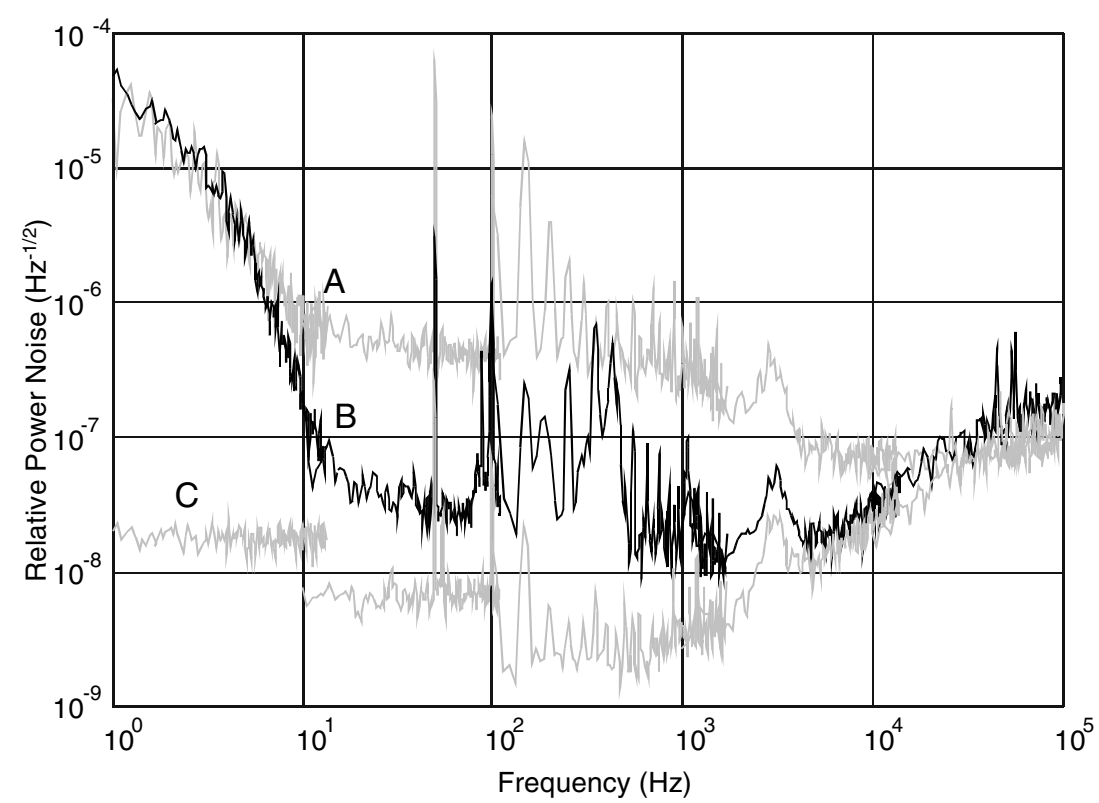

Figure 3. Relative power noise spectral density of the laser system: (A) without stabilization circuit, (B) out-of-loop signal with independent photoreceiver (not displayed in figure 1) and (C) upper limit of the in-loop signal. Below $10^{3} \mathrm{~Hz}$ the true in-loop signal is covered by the spectrum analyser input noise.

the beam splitter and the photodiodes due to their spatially slightly varying properties. For so far unknown reasons the noise reduction ceases to work below $5 \mathrm{~Hz}$. Further investigation into the latter problem as well as measures against the line noise and beam jitter are currently being carried out. All jitter-induced fluctuations in the detected power will be finally removed if the photoreceiver is located after the suspended mode cleaner in vacuum, where the beam jitter is strongly reduced.

\section{An Nd:YAG-Nd:YVO 4 system}

In order to increase the output power of the system without large modifications, an injectionlocked laser system with an Nd:YAG NPRO as the master laser and an $\mathrm{Nd}: \mathrm{YVO}_{4}$ power oscillator as the slave laser has been evaluated.

The advantages of $\mathrm{Nd}: \mathrm{YVO}_{4}$ as the gain medium over $\mathrm{Nd}$ :YAG are its anisotropic emission cross section which furthers polarized laser emission, its birefringence that suppresses depolarization losses and its lower saturation intensity which affords the application of an output coupler with higher transmission and hence reduces the detrimental effect of other resonator losses. Furthermore, the pump absorption band of $\mathrm{Nd}: \mathrm{YVO}_{4}$ at $809 \mathrm{~nm}$ is wider than that of Nd:YAG, which reduces the requirements on the pump diodes. Its general disadvantages are the low thermal conductivity and the brittleness of the material which leads to a low damage threshold with respect to pump power absorption. For end-pumped $0.5 \%$ doped rods, damage thresholds of about $58 \mathrm{~W} \mathrm{~mm}^{-2}$ have been reported [18]. This threshold can be increased by $50 \%$ by the application of composite rods with undoped end-caps [19], which reduce the temperature and the thermal stress ofthe pumped facet. A problem in 
combining Nd: $\mathrm{YVO}_{4}$ with $\mathrm{Nd}$ :YAG might be its slightly shorter centre wavelength of the gain profile at room temperature.

In order to set up an $\mathrm{Nd}: \mathrm{YVO}_{4}$ power oscillator, the general layout of the $\mathrm{Nd}$ :YAG slave laser has been left unchanged. The Nd:YAG rods were replaced by composite $0.5 \%$ doped $\mathrm{Nd}: \mathrm{YVO}_{4}$ rods. At the $450 \mu \mathrm{m}$ radius pump spots, a damage threshold of about $55 \mathrm{~W}$ was calculated. The Brewster plates were removed from the resonator. The mode size in the crystal was increased to $400 \mu \mathrm{m}$ radius in order to avoid higher mode oscillation. At the same time this increased the efficiency for the fundamental mode. At $2 \times 22 \mathrm{~W}$ pump power $22 \mathrm{~W}$ output power in a 1.1 times diffraction limited mode could be extracted through a $16 \%$ output coupler.

Despite the slightly shorter centre gain wavelength of $\mathrm{Nd}: \mathrm{YVO}_{4}$ compared to $\mathrm{Nd}: \mathrm{YAG}$ at same material temperature, the $\mathrm{Nd}: \mathrm{YVO}_{4}$ laser was easily locked to the Nd:YAG NPRO yielding more than $22 \mathrm{~W}$ single frequency output. This can be explained by a high-core temperature of the $\mathrm{Nd}: \mathrm{YVO}_{4}$ rod due to the low thermal conductivity of the material and the high thermal load. Comparing the emission of master and stand alone slave individually with respect to their crystal mount temperature ${ }^{1}$ we found the same wavelength of $1064.15 \mathrm{~nm}$ at $30{ }^{\circ} \mathrm{C}$ and tuning coefficients of $0.0063 \mathrm{~nm} \mathrm{~K}^{-1}$ for the $\mathrm{Nd}$ :YAG and $0.0030 \mathrm{~nm} \mathrm{~K}^{-1}$ for the $\mathrm{Nd}: \mathrm{YVO}_{4}$.

\section{Conclusion}

The GEO 600 injection-locked laser system approached a reliable performance that moves it from an object of research and laser development to a tool for gravitational wave research. Nevertheless there is a continuous search for further improvements, which will in future be oriented towards the qualification as a front end for a $200 \mathrm{~W}$ power stage for future gravitational wave detectors.

\section{Acknowledgment}

The authors gratefully acknowledge the project funding by the Niedersächsische Volkswagenstiftung.

\section{References}

[1] Willke B, Brozek O S, Danzmann K, Fallnich C, Goßler S, Lück H, Mossavi K, Quetschke V, Welling H and Zawischa I 2000 The GEO 600 stabilized laser system and the current-lock technique Gravitational Waves, 3rd Eduardo Amaldi Conf. (Pasadena, CA, 12-16 July 1999) ed S Meshkov (AIP Conf. Proc. 523) (Melville, New York: American Institute of Physics) p 215

[2] Freise A and the GEO 600 Team 2002 The first arm of GEO 600 Proc. of the 4th Eduardo Amaldi Conf. on Gravitational Waves (Perth, Western Australia, 8-13 July 2001) Class. Quantum Grav. 191389

[3] Siegman A E 1986 Lasers (Sausalito, CA: University Science Books)

[4] Farinas A D, Gustafson E K and Byer R L 1995 Frequency and intensity noise in an injection-locked, solid-state laser J. Opt. Soc. Am. B 12328

[5] Barillet R, Brillet A, Chiche R, Cleva F, Latrach L and Man N 1996 An injection-locked Nd:YAG laser for the interferometric detection of gravitational waves Meas. Sci. Technol. 7162

[6] Ottaway D J, Veitch P J, Hamilton M W, Hollit C, Mudge D and Munch M 1998 A compact injection-locked Nd:YAG laser for gravitational wave detection IEEE J. Quantum Electron. 342006

[7] Huntington E H, Ralph T C and Zawischa I 2000 Sources of phase noise in an injection-locked solid-state laser J. Opt. Soc. Am. B 17280

1 Be aware that due to the different geometry of both crystals and mounts a comparison of crystal temperature is not directly possible from these data. 
[8] Ralph T C, Harb C C and Bachor H-A 1996 Intensity noise of injection-locked lasers: quantum theory using a linearized input-output method Phys. Rev. A $\mathbf{5 4} 4359$

[9] Ralph T C, Huntington E H, Harb C C, Buchler B C, Lam P K, McClelland D E and Bachor H-A 1999 Understanding and controlling laser intensity noise Opt. Quantum Electron. 31583

[10] Kane T J and Byer R L 1985 Monolithic, unidirectional single-mode Nd:YAG ring laser Opt. Lett. 1065

[11] Freitag I, Tünnermann A and Welling H 1995 Power scaling of monolithic miniature Nd:YAG ring lasers to output powers of several watts Opt. Commun. 115511

[12] Harb C C, Gray M B, Bachor H-A, Schilling R, Rottengatter P, Freitag I and Welling H 1994 Suppression of the intensity noise in a diode-pumped neodymium:YAG nonplanar ring laser IEEE J. Quantum Electron. 30 2907

[13] Bondu F, Fritschel P, Man C N and Brillet A 1996 Ultrahigh-spectral-purity laser for the VIRGO experiment Opt. Lett. 21582

[14] Muscha M, Telada S, Nakagawa K, Ohashi M and Ueda K 1997 Measurement of frequency noise spectra of a frequency-stabilized LD-pumped Nd:YAG laser by using a cavity with separately suspended mirrors $O p t$. Commun. 140323

[15] Willke B, Brozek S, Danzmann K, Quetschke V and Gossler S 2000 Frequency stabilization of a monolithic Nd:YAG ring laser by controlling the power of the laser-diode pump source Opt. Lett. 251019

[16] Zawischa I, Brozek O S, Quetschke V, Fallnich C, Willke B, Danzmann K and Welling H 2000 GEO 600 slave laser prototype II Gravitational Waves, 3rd Eduardo Amaldi Conf. (Pasadena, CA, 12-16 July 1999) ed S Meshkov AIP Conference Proceedings 523 (Melville, New York: American Institute of Physics) p 389

[17] Weast R C (ed) 1989 CRC Handbook of Chemistry and Physics 70th edn (Boca Raton, FL: CRC Press)

[18] Chen Y-F 1999 Design criteria for concentration optimization in scaling diode end-pumped lasers to high powers: influence of thermal fracture IEEE J. Quantum Electron. 35234

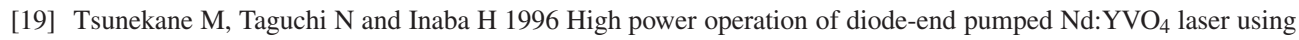
composite rod with undoped end Electron. Lett. 3240 